\title{
Señderes
}

\section{Estudiar prácticas letradas escolares con el apoyo de intérpretes en lenguas indígenas de México'}

\author{
Study school letrated practices with the support \\ of interpreters of México indigenous languages
}

\begin{abstract}
Autores
Amanda Cano Ruíz ${ }^{2}$

Verónica Aguilar Martínez ${ }^{3}$

Recibido: 12 de mayo de 2017.

Aprobado: 30 de noviembre de 2017.
\end{abstract}

\footnotetext{
1 Producto final de investigación del Doctorado en Investigación Educativa cursado en el Instituto de Investigaciones en Educación de la Universidad Veracruzana, adscrito a la Línea de investigación: Lengua escrita, adquisiciones prácticas y usos del Instituto de Investigaciones en Educación de la Universidad Veracruzana, y asesorado por el doctor Jorge E. Vaca Uribe. Inició en agosto de 2011 y culminó en marzo de 2014.

2 Licenciada en Educación Primaria por la Benemérita Escuela Normal Veracruzana Enrique C. Rébsamen. Magíster en Educación y doctora en Investigación Educativa de la Universidad Veracruzana, México. mandy_caru@hotmail.com 3 Licenciada en Lengua y Literatura Hispánica de la Universidad Veracruzana, México. Profesional vinculada al Centro de Investigación en Lengua Escrita y Matemáticas. veronica.aguilar.mtz@gmail. com
}

\section{Resumen}

Se analiza la forma en que intérpretes de una lengua indígena contribuyeron al desarrollo de una investigación etnográfica ubicada principalmente en una escuela secundaria donde los estudiantes usan la lengua popoluca de la región de la Sierra de Santa Marta, en el estado de Veracruz, México. El objetivo del estudio fue analizar cómo se apropiaban de prácticas letradas en la asignatura de español. El alcance dependió del trabajo realizado por intérpretes como mediadores lingüístico-culturales que facilitaban la comprensión mutua entre investigador y estudiantes. Se detalla el proceso de selección de intérpretes, las estrategias de trabajo desarrolladas y la información inicial que brindaron al investigador. Se profundiza en la participación de una intérprete en las clases de español y en el seguimiento de tareas extraescolares. Igualmente, se reconocen estrategias de trabajo conjunto que permitieron identificar el papel central de la lengua indígena en la apropiación de la lengua escrita en español. 
Palabras clave: etnografía, educación secundaria, lengua indígena, clases de español, interpretación lingüística cultural.

\section{Abstract \\ It is analyzed the way in which interpreters in an indigenous language contributed to the development of an ethnographic research located in a secondary school whose students use the Popoluca language of the region of Sierra de Santa Marta, in the state of Veracruz, Mexico. The objective of the study was to analyze the appropriation of literate practices in the subject of Spanish. Its scope depended on the work performed by interpreters as linguistic- cultural mediators in facilitating mutual}

understanding between researchers and students. It details the process of selection of the interpreters, the work strategies developed and the initial information that they provided the researcher. The participation of an interpreter in the Spanish classes and in the follow-up of extracurricular tasks is deepened.

We recognize diverse strategies of joint work that allows to identify the central role of the indigenous language of the students in the appropriation of the Spanish written language.

Key words: ethnography, high school, indigenous language, Spanish classes, cultural linguistic interpretation.

\section{Introducción}

En México, las investigaciones educativas realizadas en contextos indígenas con vitalidad lingüística representan un campo fértil y que requiere ser explorado. Algunos autores que han tenido acercamiento a esta línea de estudio son: Bertely (2005), Rockwell (2006 y 2008), Hamel (2003), Podestá (2003) y Jiménez (2011). Cuando el investigador se adentra en estos espacios enfrenta retos importantes, de los cuales el principal es dominar la lengua de los pobladores. Puede darse el caso de que, como se documenta aquí, el investigador no domine la lengua requerida y deba apoyarse en intérpretes, de modo que se conforma un equipo de trabajo.

La investigación que enmarca este artículo nació con un interés por conocer la forma en que se utilizaba, en escuelas secundarias del estado de Veracruz, la tecnología distribuida por la estrategia Habilidades Digitales para Todos (HDT), en particular los Objetos de Aprendizaje (OdA) diseñados como apoyo para la enseñanza del español. ${ }^{4}$ Se sabía que en la zona de estudio estaban presentes dos lenguas indígenas: el náhuatl y el popoluca de la Sierra. ${ }^{5}$ Sin embargo, en nuestro país la educación intercultural bilingüe opera en los dos niveles educativos previos: preescolar y primaria, y se establece que en la educación secundaria el currículum se desarrolla en español, por lo que la situación lingüística no fue motivo de preocupación.

4 Este proyecto nacional buscaba impulsar el desarrollo y uso de las Tecnologías de la Información y la Comunicación (TIC) en las escuelas de educación básica de todo el país.

5 Según el Censo de Población y Vivienda 2015, en México 7.382.785 personas de 3 años y más hablan alguna lengua indígena. El náhuatl es la de mayor número de hablantes en diversas entidades del país (1.725.620) y el popoluca de la Sierra, con presencia solo en Veracruz, es hablado por 37.707 individuos. 
En la primera fase del estudio participaron tres escuelas: una telesecundaria de contexto popoluca, otra de contexto náhuatl y una escuela secundaria general de contexto urbano. Debido al escaso uso que dieron al equipamiento de HDT, se decidió enfocar la atención en las clases de español. Se identificó que, en las telesecundarias, los estudiantes usaban dos lenguas en las sesiones: la indígena y el español. Este escenario condujo a delimitar que la unidad de análisis serían las clases de español y el trabajo de campo se profundizaría en la telesecundaria de contexto popoluca, pues en ella se presentaba la mayor riqueza de interacciones entre estudiantes en la lengua indígena.

El avance en el trabajo de campo y el análisis de los datos permitieron establecer como objetivo principal del estudio analizar cómo se apropiaban los estudiantes de las prácticas letradas promovidas por la escuela desde la asignatura de español. Los referentes teóricos en los que nos basamos para su abordaje provienen de la didáctica de las lenguas (Dolz, Gagnon y Mosquera, 2009), la sociología de la lengua (Lahire, 2010), la lingüística (Catach, 1996) y los aportes de Cummins (2002) sobre contextos educativos bilingües. Se vivió un proceso de transformación del investigador, característico de los estudios etnográficos (Rockwell, 2009). En este recorrido se contó con la colaboración de intérpretes, quienes fueron fundamentales en el conocimiento de la realidad estudiada.

De esta manera, el objetivo que nos proponemos en el artículo es documentar el trabajo colaborativo desarrollado entre un grupo de investigación conformado por un etnógrafo y los intérpretes de una lengua indígena, en cuya metodología resaltan los procedimientos seguidos, los retos enfrentados, los aprendizajes construidos, así como la mediación lingüística-cultural que se generó. Se destaca en particular la intérprete que acompañó al investigador en dos fases intensivas de trabajo de campo en la telesecundaria de contexto popoluca, razón por la cual se ahondará más en este caso.

Se parte de la revisión de algunos elementos teóricos relativos a los conceptos de traducción, interpretación y mediación. Enseguida se puntualizan aspectos relativos a la metodología del estudio, y se profundiza en las diversas fases desarrolladas y la participación de los intérpretes en ellas. Posteriormente se presentan y analizan algunos de los principales resultados obtenidos a partir del trabajo colaborativo desarrollado.

\section{Traducir, interpretar y mediar}

La traducción y la interpretación hacen posible el contacto entre diversas culturas y sociedades. Según Jiménez-Ivars (2002), la traducción permite reformular un mensaje expresado originalmente en una lengua específica (lengua de partida) en otra lengua (lengua de llegada). Añade que la interpretación, por su parte, es una especie de traducción que se desarrolla de manera oral, ya sea en la modalidad simultánea (al mismo tiempo que la enunciación del texto origen) o en la consecutiva 
(inmediatamente después). Por sus usos, también se distingue entre interpretación social, de tribunales, de conferencia, entre otras. Lo que tienen en común todas las clases de interpretación es que permiten la comprensión contextualizada de significados, generalmente lingüísticos.

Existen diversas maneras de clasificar el trabajo desarrollado por los intérpretes, aquí se hace referencia a un tipo particular que se engloba dentro de lo cultural. A lo largo de esta investigación se advirtió que el trabajo de los intérpretes se acercaba a la mediación lingüístico-cultural, término con el que se hace referencia a la labor de un intérprete en contextos pluriétnicos o multiculturales (Sales, 2003), donde se busca facilitar el reconocimiento y el acercamiento entre los significados expresados por participantes con diferencias culturales marcadas. Esta mediación pretende lograr una mejor comunicación no solo lingüística, sino también cultural, y una mutua comprensión entre terceros.

Giménez (1997) explica que la mediación lingüístico-cultural tiende puentes entre personas que se involucran en relaciones interétnicas, y enfatiza en que se ubica en situaciones específicas donde las diferencias socioculturales de los sujetos claramente son centrales o influyentes:

En dichas situaciones los actores sociales en interacción (individuos, grupos, organizaciones, comunidades, etc.) están dando importancia considerable consciente o inconscientemente - a la diferenciación del Otro o a la propia respecto del Otro, en términos físicos, de conducta, de modo de vida, de lenguaje, de simbolización, de expresión de valores, etc. (Giménez, 1997, p. 133).

Es decir, se trata de sujetos con una identidad etnocultural diferenciada que condiciona las relaciones que puedan llegar a establecer. En el caso que nos ocupa los sujetos son: un investigador que pertenece a una mayoría lingüística en México, y un grupo de estudiantes (con sus familias) que forman parte de una minoría lingüística en el país.

La mediación lingüístico-cultural es una actividad compleja. El mediador debe tener claro el objetivo del acercamiento entre las dos partes, además de contar con un bagaje cultural que le permita comprender ambas lógicas culturales en presencia (Giménez, 1997). Es ideal contar con un mediador bicultural (con conocimiento de las dos culturas involucradas), aspecto que se buscó cumplir en esta investigación.

\section{Metodología}

En este apartado se describirá la metodología general desarrollada en la investigación y se enfatizará en el papel desarrollado por los intérpretes en las diversas fases que contempló el estudio. Se trató de un proceso de investigación inductivo, a lo largo de cuatro fases de trabajo de campo. 


\section{Primera fase}

Esta se desarrolló de octubre de 2010 a enero de 2011 y durante ella se visitaron de manera simultánea las tres escuelas participantes. Se realizaron entrevistas informales con directivos, docentes y estudiantes, y se contó con el acceso a los archivos escolares de donde se obtuvieron datos históricos de las instituciones. También se documentaron rasgos generales de las localidades. Se observaron dos clases de español, sin apoyo en las TIC, de los tres docentes seleccionados en las escuelas. En cuanto a la trayectoria de uso del equipamiento de HDT, hubo reuniones del personal docente para llegar a acuerdos sobre el uso del aula de cómputo, dos clases en el aula de cómputo coordinadas por el director de la telesecundaria de contexto popoluca y seis clases de español donde se usaban objetos de aprendizaje de HDT en la secundaria general. Todo esto derivó en la construcción de registros de campo y transcripciones de las clases que, en el caso de las telesecundarias, estaban incompletas, pues había diálogos de los estudiantes en su lengua materna a cuyo contenido no se podía acceder.

Esta situación fue la que motivó la decisión de involucrar intérpretes en la investigación. Inicialmente se pensó en contar con intérpretes profesionales, pues se trata de una actividad que demanda una formación específica en este campo (Márquez, 2011). A fin de contar con orientación sobre intérpretes en el estado, se visitó la instancia estatal responsable de estudiar, difundir y preservar las lenguas indígenas de Veracruz, la Academia Veracruzana de Lenguas Indígenas (AVELI), pero contestaron que carecían de esa información. Posteriormente, hubo un acercamiento a los estudiantes de la Licenciatura en Gestión Intercultural de la Universidad Veracruzana Intercultural (UVI), Sede Selvas, que desde 2005 se ubica cerca de la zona de estudio. En esta universidad estudian algunos jóvenes hablantes de las lenguas indígenas de la región. Dentro de la carrera pueden optar por la Orientación Lenguas que, según el programa de estudios, a su egreso les permitirá poder "intervenir en procesos comunicativos interlingüísticos enmarcados en un enfoque intercultural” (UVI, 2007, p. 14). Después de una serie de visitas, se logró iniciar una colaboración con Emilia Jiménez López, estudiante de séptimo semestre, bilingüe en español y popoluca de la Sierra, y con Rosaura Bautista Bautista, de quinto semestre, bilingüe en español y náhuatl. Con las estudiantes se compartieron las transcripciones de las clases de español, audios y videos, donde los estudiantes dialogaban en lengua indígena. Ellas realizaron la revisión detallada de estos materiales y la interpretación correspondiente.

\section{Segunda fase}

Esta se llevó a cabo de febrero a mayo de 2011, tuvo como propósito principal profundizar en el desarrollo de las clases de español en la telesecundaria seleccionada. Se tuvo acceso a las aulas de los cuatro docentes de la escuela y se observaron 12 clases de español en total, tres de cada profesor. También se desarrollaron entrevistas formales e informales con cada uno de los docentes, el director y padres de familia de la localidad. De nuevo se construyeron registros ampliados de estos eventos y posteriormente descripciones de cada una de las clases donde se señalaban los momentos en los cuales los estudiantes hablaban en popoluca. 
La estancia en la zona de estudio y la colaboración con un proyecto de orden ambiental de la Universidad Veracruzana $^{6}$ (UV) facilitó, en esta etapa del estudio, la participación del intérprete Rubén Mateo Gutiérrez, técnico de campo en fauna y flora silvestre de esta institución. Él era habitante de una comunidad popoluca y mostró disposición y experiencia para este tipo de actividades. Esta persona transcribió y tradujo algunos fragmentos de audios y videos de clases de español, pues le era imposible reconstruir todas estas interacciones y surgían muchas interrogantes acerca de lo sucedido, ya que los videos y audios no le brindaban toda la información que requería, con el fin de conocer en mayor detalle el contenido de las conversaciones de los estudiantes.

Esto permitió reconocer la importancia de contar con un intérprete que colaborara con el investigador durante los eventos escolares y extraescolares en la comunidad y la telesecundaria seleccionada. Era importante adentrarse en la comprensión de la dinámica de las clases (cuándo, por qué y para qué empleaban la lengua materna). Además, como se identificó que muchas de las actividades del currículum oficial eran desarrolladas como tareas fuera de la escuela, era central conocer cómo las enfrentaban los estudiantes en sus hogares y así responder a las exigencias escolares.

Inicialmente, el técnico de campo de la Universidad Veracruzana manifestó interés en desempeñar esta tarea, pero sus compromisos profesionales se lo impidieron. Se invitó a la estudiante de la UVI, Emilia Jiménez López, quien aceptó participar, aunque muy cerca del reinicio del trabajo de campo desistió. Ella recomendó a Julieta Martínez Hernández, una joven que había colaborado como intérprete en dependencias de salud en una localidad vecina de donde se ubicaba la telesecundaria. Se dio un acercamiento con ella y decidió participar.

\section{Tercera fase}

Esta fase se desarrolló de octubre a diciembre de 2011. En ella se observaron ocho clases de español y cinco tareas extraescolares de 16 estudiantes de primer grado que eran atendidos por una docente. También se hicieron entrevistas informales a la docente, estudiantes y padres de familia.

Con relación al trabajo desarrollado con la intérprete, es importante aclarar que de manera previa a la fase se realizaron reuniones para tomar acuerdos. Desde el principio se asumió que la intérprete y el investigador aprenderían a colaborar juntos. Era necesario construir estrategias de trabajo, pues tampoco se tenía acceso a investigaciones que documentaran una experiencia parecida a la que se estaba viviendo (colaboración intérprete-investigador en una etnografía de aula). Se trató de un trabajo inductivo y constructivo que tuvo como centro comprender mejor a los sujetos implicados en la investigación, dentro de los márgenes que brinda el enfoque etnográfico.

6 El proyecto se titula "Influencia de variables socioeconómicas sobre el uso de suelo en áreas de vegetación primaria en la Sierra de Santa Marta, Veracruz, y su impacto en las poblaciones de primates y mamíferos medianos”, del Instituto de Neuroetología de la Universidad Veracruzana. 
Estas reuniones de trabajo fueron valiosas para el conocimiento mutuo y llegar a acuerdos. Se dialogó en torno al objetivo de la investigación y a partir de ahí se aclararon las expectativas sobre las tareas para desarrollar. Se buscó comunicar, en términos muy simples, que el interés central de estar en la escuela era comprender, por un lado, las expectativas y exigencias académicas que la telesecundaria les demanda a los estudiantes, y las versiones que los docentes construían de las prácticas de lengua escrita; por otro lado, las dificultades que presentaban los estudiantes para participar en los eventos letrados, así como las estrategias o recursos lingüísticos y culturales que ponían en juego para superarlas.

Se comentó sobre los eventos en los que generalmente se participaba dentro y fuera de la escuela: clases de español, reuniones formales e informales con docentes y entrevistas a estudiantes, docentes y padres de familia. También se habló del seguimiento a las tareas extraescolares de la asignatura de español, lo que implicaba incursionar en nuevos escenarios dentro de la localidad, donde se esperaba un uso más intensivo del popoluca.

Se aclaró el tipo de relación que se debía entablar con los sujetos de la investigación: el objetivo no era evaluar el trabajo de los profesores, los jóvenes y sus familias sino comprender sus formas de participación alrededor del trabajo propuesto por la escuela en la asignatura de español. Ello demandaba una actitud abierta ante sus puntos de vista y una escucha comprensiva de sus interacciones. Se conversó acerca del tiempo que había llevado ganar cierto grado de confianza y la importancia de seguir avanzando en ese sentido. En estos diálogos se advirtió que la intérprete conocía a algunos de los estudiantes y a sus familias, lo que se vio como una ventaja para el trabajo que desarrollaría.

También se abordó lo relativo a la construcción de notas de campo, una práctica nueva para esta intérprete, pues estaba habituada a la traducción simultánea, sin apoyo en la escritura. Se le proporcionó una libreta y se le explicó el proceso de construcción de notas y registros de campo, a partir de algunos ejemplos elaborados por el investigador al observar las clases.

Se comentó con la intérprete que, en el caso de las clases de español, las notas partían de un encabezado, recuperaban la secuencia de actividades propuesta por el profesor y la forma en que los estudiantes participaban en ellas, y registraban los momentos en que usaban el popoluca. Las notas se convertían en registros ampliados, una vez revisado el video de la clase ${ }^{7}$. Aclaramos que la toma de notas no siempre se realizaba durante la observación. En ocasiones el etnógrafo, por considerarlo pertinente, prefería concentrar su atención sólo en observar o dialogar con las personas y recuperar estos encuentros posteriormente, haciendo uso de su memoria. Es decir que horas después se redactaban en computadora las aproximaciones de lo que las personas habían

\footnotetext{
7 En la investigación se diferenció entre notas de campo (los escritos a mano que se redactaban al observar una clase, realizar una entrevista, etc.), registros del trabajo de campo (ampliación de las notas de campo) y diario de campo, orientado al registro de aspectos reflexivos por parte del investigador.
} 
expresado oralmente. Quedó claro que la forma de tomar notas se afinaría al entrar al campo; ahí se probaría y valoraría cuál estrategia era más conveniente para el registro de las intervenciones de los estudiantes.

Las reuniones iniciales con la intérprete sirvieron también para familiarizarla con el uso de las grabadoras de audio y las cámaras de video y fotografía, que serían apoyos importantes en la ampliación de sus notas de campo.

Las clases de español fueron los principales eventos en que participó la intérprete. Ella se ubicó al fondo del aula para tener un panorama de la dinámica de las clases e identificar a los estudiantes, así como sus intervenciones y diálogos en popoluca. Esta tarea se complicaba por la forma en que los estudiantes usaban su lengua: entablaban pequeños diálogos en voz baja y hablaban varios al mismo tiempo sobre diferentes aspectos. Había mucha más interacción en el aula que la percibida previamente por el investigador. Al momento de revisar las notas de campo que construyó, se corroboró que eran de difícil comprensión incluso para ella, como se puede observar en la Figura 1.

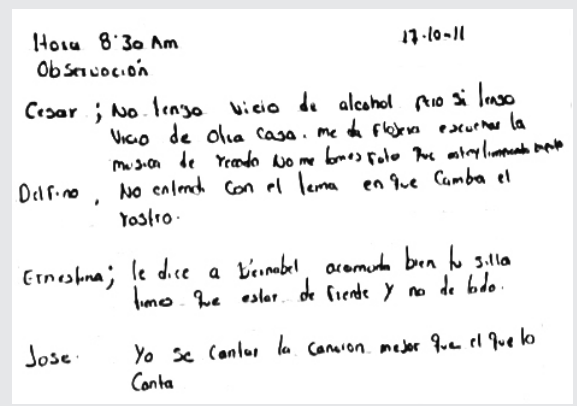

Hora 8:30 a.m

Observación

Cesar; No tengo vicio de alcohol pero si tengo vicio de otra cosa, me da flojera escuchar la música de recodo. No me tomes foto que estoy limpiando zapato

Delfino; No entendí con el tema en que cambia el rostro.

Ernestina; le dice a Bernabe acomoda bien tu silla tienes que estar de frente Y no de lado.

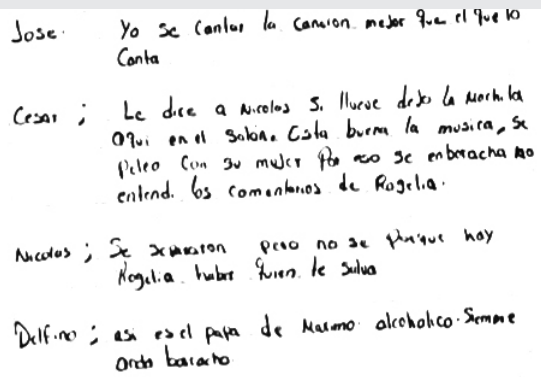

José; Yo se cantar la canción mejor que el que lo canta.

Cesar; Le dice a Nicolás Si llueve dejo la Mochila aquí en el salón. Esta buena la música, se peleo con su mujer Por eso se emborracha no entendí los comentarios de Rogelia.

Nicolas; Se separaron pero no se porque hay Rogelia haber quien te salva

Delfino; así es el papa de Máximo. Alcohólico. Siempre anda borracho

Figura 1. Primeras notas de campo construidas por la intérprete.

Fuente: elaboración propia 
En la figura anterior vemos cómo las notas incluyen segmentos de diálogos entre diferentes estudiantes, sin un contexto. Investigador e intérprete dialogaron acerca del sentido de la investigación y el contenido esperado de las notas: descripción de las actividades generales de los estudiantes y su profesor, incluyendo los intercambios en popoluca, con datos del alumno que intervenía y la hora aproximada en que lo hacía. También se seleccionó a determinados estudiantes, parejas o equipos del grupo (dependiendo de la dinámica de clase) para interpretar en detalle sus interacciones en popoluca; la intérprete tomaría grabaciones que después escucharía para ampliar sus notas.

La intérprete dividía su cuaderno de notas en tres columnas: en la primera especificaba el tiempo (hora y minutos); en la segunda, las actividades generales que el docente iba proponiendo a los estudiantes a lo largo de la clase, y en la tercera registraba los comentarios que algunos estudiantes hacían en popoluca alrededor de ellas (sin que fuera textual). Una vez terminada cada clase, y fuera de la escuela, escuchaba las grabaciones para ampliar la traducción de las expresiones de los estudiantes. Posteriormente se generaba un diálogo investigador-intérprete alrededor de las notas de campo que ambos habían tomado.

En estos espacios de intercambio con el investigador, la intérprete se interesaba en narrar lo que a su juicio había sucedido en las clases y la forma en que los estudiantes habían interpretado las actividades propuestas (por el profesor o por los materiales curriculares). Comentaba también las dificultades de los jóvenes y la forma en que las solucionaban. Explicaba su punto de vista sobre el papel que cumplía el popoluca en todo este proceso: cuándo recurrían a él, por qué lo hacían y sobre qué temas dialogaban.

Estos espacios de intercambio fueron importantes para el avance en la comprensión del fenómeno estudiado. Por un lado, se hizo evidente que la intérprete se identificaba con los jóvenes. Contó que ella había vivido algo similar (una década atrás), cuando estudió en una telesecundaria cercana. Sus experiencias le permitían hacer comentarios pertinentes sobre lo que estos jóvenes estaban enfrentando al participar en clases en las que tanto los docentes como los materiales de estudio empleaban una lengua que ellos no dominaban. Es importante agregar que, al momento de las observaciones de clase, la misma intérprete tuvo dificultades para comprender algunas expresiones de los docentes y de los libros de texto de español de primero y segundo grado.

Por otro lado, en muchas ocasiones la intérprete también colaboró para que el investigador comprendiera el español local empleado por los estudiantes y sus familias; estaba asumiendo un rol de mediadora lingüístico-cultural, pues se preocupaba por lograr que el investigador interpretara adecuadamente los usos locales, tanto lingüísticos como de forma de vida. Además, se notaba que cada vez tenía más claros los intereses de la investigación y la forma en que podría brindar un apoyo pertinente. Es así que se empezó a desarrollar un trabajo colaborativo: durante las clases, en ocasiones juzgaba pertinente informarle al investigador del contenido de 
los diálogos de los estudiantes, a fin de saber si hacía falta plantearles alguna pregunta en su lengua (interpretación consecutiva). Otras veces era el investigador quien se acercaba a la intérprete para conocer el contenido de ciertas intervenciones de los estudiantes, las cuales en la mayoría de las ocasiones trataban sobre cómo solucionar los ejercicios de los libros de texto. El investigador registraba esta información, y al final del día construía lo que en etnografía se conoce como registros de trabajo de campo.

La intérprete participó en la negociación del acceso a los domicilios de los estudiantes para el seguimiento de las tareas extraescolares, sirvió de apoyo en la solicitud de los permisos a los familiares de los jóvenes, explicaba el motivo de la visita y justificaba el uso de cámaras y grabadoras. Indudablemente, el hecho de que hablara popoluca generaba confianza y facilitaba que las madres (principalmente) expresaran sus dudas sobre el seguimiento a las tareas de sus hijos. La mayoría de estas tareas implicaron trabajo en pequeños equipos (de dos a cuatro miembros). La intérprete se centró en registrar el tiempo, las actividades desarrolladas y los diálogos de los estudiantes en lengua indígena. En ocasiones se acercaba al oído del investigador para traducirle las dudas que en ese momento presentaban los jóvenes. Esto podía conducir al desarrollo de entrevistas informales a los estudiantes para mejorar la comprensión del evento; dichas entrevistas se desarrollaban alternando español y popoluca, pues algunas veces los jóvenes preferían hablar en popoluca. Entonces la intérprete los escuchaba y planteaba nuevas preguntas que los jóvenes respondían con fluidez y libertad. Todos estos eventos también fueron grabados en audio y video.

\section{Cuarta fase}

Esta fase se llevó a cabo entre marzo y abril de 2012; en ella se observaron seis clases de español de una profesora a un grupo integrado por 19 estudiantes de segundo grado y nueve tareas extraescolares, también se realizaron entrevistas informales con la docente, los estudiantes y padres de familia. La intérprete participó en todos los eventos observados en la escuela, lo que abarcó nuevamente clases de español y tareas extraescolares.

\section{Análisis de datos}

En esta investigación se realizaron diversos textos analíticos a lo largo de todo el trabajo de campo. Al finalizar la última fase, se intensificó este proceso. Se contaba con una importante cantidad de eventos registrados en donde se hacía evidente la alternancia de estas dos lenguas. Por motivos de análisis más detallado, fue necesario transcribir algunos de estos eventos con la traducción de los momentos en que los estudiantes usaban la lengua indígena. Esta tarea requirió también el trabajo conjunto de investigador e intérprete (o, en este caso, de dos intérpretes). A grandes rasgos, el proceso que se siguió fue el siguiente: 
- El investigador transcribió cuatro eventos: tres tareas extraescolares y una clase de español, que resultaban de importancia para la investigación, y allí señaló los momentos en que los estudiantes hacían uso de la lengua indígena. Compartió estas transcripciones con la intérprete, a fin de familiarizarla con el código de transcripción empleado y especificar en cuáles apartados requería una traducción.

- La intérprete llevó a cabo una serie de ejercicios con el apoyo del investigador. En ellos se fue aclarando el nivel de detalle y la fidelidad requeridos en la traducción de las expresiones de los estudiantes.

- La intérprete realizó de manera autónoma las traducciones necesarias, que fueron objeto de revisión por el investigador y un segundo intérprete a fin de cuidar el rescate de las ideas expresadas por los jóvenes.

Fue difícil para la intérprete apropiarse del código de transcripción propuesto. Además, al revisar las traducciones, se observó la presencia de diversos errores sintácticos, ortográficos y semánticos, que se corrigieron. Su español escrito, como el de los estudiantes de telesecundaria, reflejaba cierta influencia del popoluca oral, cuestión que resultó muy interesante para la investigación, pero de difícil manejo para la tarea de reflejar fielmente lo expresado por los jóvenes. Al respecto, se reconoce lo complejo de construir traducciones de la oralidad aun por expertos en la materia, se habla de que ello constituye "un problema general de traducción (general y objetivable, en terminología de Nord, 1997, p. 64)” (García, 2004, p. 125). Cabe mencionar que todas estas vicisitudes se superaron y, después de varios meses, se logró construir una versión mejorada de las transcripciones.

Se advirtió que las transcripciones-traducciones de los eventos seleccionados no reflejaban la intensidad con que los jóvenes transitaban por las dos lenguas. Éstas se diferenciaban solo por los colores utilizados: uno para las expresiones originalmente en español y otro para las traducidas del popoluca. Se decidió que era importante escribir las intervenciones de los estudiantes en lengua indígena para representar de forma más clara cómo construyen múltiples puentes entre el español, tanto oral como escrito, y el popoluca oral. Para esta tarea se solicitó apoyo al intérprete participante con mayor dominio del popoluca escrito (el técnico de campo de la Universidad Veracruzana). Nuevamente fue necesario un trabajo colaborativo investigadorintérprete, ya que se presentaron dificultades para la escritura del popoluca, que se solucionaron gracias a la consulta de materiales bibliográficos (principalmente libros sobre gramática del popoluca y un diccionario del popoluca de la Sierra). ${ }^{8}$ Además se contó con el apoyo de un lingüista experto en el estudio de esta lengua en México. Esto permitió contar con transcripciones completas de estos eventos.

8 Rastreamos algunos materiales en popoluca de la Sierra editados por la Secretaría de Educación Pública (SEP), el Instituto Lingüístico de Verano, el Instituto Nacional de Lenguas Indígenas (INALI) y la Academia Veracruzana de Lenguas Indígenas (AVELI). Todos fueron un importante apoyo para el trabajo de transcripción realizado por el intérprete. 


\section{Resultados y discusión}

La investigación permitió reconocer que el proceso de apropiación de prácticas letradas escolares se da en el contexto de una tensión muy fuerte entre las características culturales y lingüísticas de los estudiantes y lo que los docentes proponen como objeto de enseñanza. Las prácticas letradas escolares propuestas por la escuela provienen de grupos o comunidades de práctica muy distantes a los referentes culturales de los jóvenes que estudian en la telesecundaria. Hay una imposición de literacidades dominantes o cultas que se derivan de lo propuesto por el propio currículum formal y que son reinterpretadas o transpuestas internamente (Dolz, Gagnon y Mosquera, 2009) por los docentes. A estos últimos les es complejo poder conectar lo propuesto por la Secretaría de Educación con la vida comunitaria de los estudiantes, y ello nos indica que el proceso de apropiación conlleva una serie de negociaciones y resignificaciones de estas prácticas como objetos de enseñanza en la escuela.

Con relación a las dificultades que enfrentan los estudiantes en este proceso, se pudo identificar que estaban aprendiendo a hablar español en un contexto académico. La variante del español que ellos manejan dista mucho del lenguaje que emplean los materiales curriculares, como expresa Cummins (2002), refiriéndose al bilingüismo: "hay una diferencia cualitativa entre las destrezas y tácticas comunicativas que los niños encuentran efectivas para satisfacer sus necesidades y alcanzar sus objetivos sociales, y aquellos que son necesarios para un rendimiento académico escolar exitoso" (p. 211). Sobre este mismo aspecto, Lahire (2010) argumenta que la escuela construye una relación específica con el lenguaje, "suponiendo que éste sea apartado, objetivado y estudiado como tal desde múltiples puntos de vista (fonológico, lexical, gramatical, textual)" (p. 123); él ha detectado que hay estudiantes que no logran entrar en estos "juegos de lenguajes escolares", lo que suele explicar por qué forman parte del fracaso escolar.

En este marco se documentó que el popoluca de la Sierra, su lengua materna, constituye una herramienta fundamental en su apropiación del español escrito, a diferencia de la concepción de los docentes de la escuela, quienes lo visualizan como el principal obstáculo para que ellos aprendan español. Gracias al trabajo realizado entre investigador e intérpretes se identificó cómo los estudiantes parten de discutir en popoluca oral una idea (incluyendo préstamos del español), para después construir una expresión en español oral con miras a trasladarla al español escrito. Esta tarea, a su vez, puede desatar nuevas discusiones en popoluca oral y ajustes en la expresión que están construyendo en español oral, para finalmente plasmarla por escrito (como diría Catach [1996], entre el lenguaje oral y escrito hay más una relación de complementariedad que de jerarquía, y más en este caso en que interviene el español como L2 o segunda lengua).

Para ilustrar este uso del popoluca de los estudiantes alrededor del aprendizaje del español escrito, se muestra en la Tabla 1 un fragmento del desarrollo de una tarea 
extraescolar (de escritura) entre dos estudiantes, Ernesto y Ramiro, de segundo grado de secundaria:

Tabla 1. Fragmento de transcripción del desarrollo de una tarea extraescolar*

\begin{tabular}{|c|c|}
\hline $\begin{array}{l}\text { E: ¿Tyi yukmi tan watp'a j'em dibujo?, ¿ji } \\
\text { tan watp'a? } \\
\text { R: iQuién sabe! } \\
\text { E: La maestra nim iga tan chiiñ tum plus } \\
\text { (R no le contesta, y E le pregunta acerca } \\
\text { de lo que tienen que escribir). ¿Jutypik } \\
\text { i watyajp'a?, ken dya a kun'ukp'a jix’i } \\
\text { jutypik tan watp'a. } \\
\text { R: Dya an jodong, wiap ta jixi kumongp'a } \\
\text { jesik tan jaykk'amum. } \\
\text { E: (busca el texto modelo en su Libro de } \\
\text { español) Yip wiaptym takuyooxap. } \\
\text { R: Wiaptyim tan waga wat. Sip } \\
\text { tan ak'kam'ayp'a yip tema yippam } \\
\text { (señalando la hoja blanca). } \\
\text { E: ken nim ig'a tum idyik (se refiere a que } \\
\text { sólo es un tema). } \\
\text { R: Junim. ¿Juppik tema? } \\
\text { E: J'em tema je'e... la experiencia de la } \\
\text { música. } \\
\text { E: ¿Tan watp'a con mayúscula? } \\
\text { R: ¿Wiaps'i? } \\
\text { E: Numas yip (se refiere a que nada } \\
\text { más el tema va escrito con mayúscula). } \\
\text { ¿Jutypiggam ity j'em libro? } \\
\text { R: Idung tyig'a jesik an mayp'a an ixp'a } \\
\text { tum cuadro }\end{array}$ & $\begin{array}{l}\text { : ¿En qué vamos hacer el dibujo?, ¿si lo } \\
\text { hacemos? } \\
\text { R: iQuién sabe! } \\
\text { E: La maestra dijo que hay que dar un } \\
\text { plus (R no le contesta, y E le pregunta } \\
\text { acerca de lo que tienen que escribir). } \\
\text { ¿Cómo lo vamos hacer?, no tengo idea de } \\
\text { cómo hacerlo. } \\
\text { R: No sé, es que puede que nos } \\
\text { equivoquemos al escribir. } \\
\text { E: (busca el texto modelo en su libro de } \\
\text { español) Éste también puede ayudarnos. } \\
\text { R: También lo podemos hacer juntos. } \\
\text { Ahora le ponemos el tema a esta } \\
\text { (señalando la hoja blanca). } \\
\text { E: Parece que dijo que nada más era uno } \\
\text { (se refiere a que sólo es un tema). } \\
\text { R: Sí. ¿Cuál es el tema? } \\
\text { E: El tema es... la experiencia de la } \\
\text { música. } \\
\text { E: ¿Lo hacemos con mayúscula? } \\
\text { R: ¿Si se puede? } \\
\text { E: Nada más este (se refiere a que nada } \\
\text { más el tema va escrito con mayúscula). } \\
\text { ¿Cómo está en el libro? } \\
\text { R: No sé, porque siempre que leo veo que } \\
\text { es un cuadro. }\end{array}$ \\
\hline
\end{tabular}

* Para abreviar, en las transcripciones se usó E para Ernesto y R para Ramiro. En la columna de la izquierda se presentan los diálogos expresados en popoluca (con algunos préstamos del español); y en la segunda columna se presentan sus traducciones. Las comillas indican lectura; lo subrayado, escritura; y los paréntesis, comentarios y precisiones del investigador.

Fuente: elaboración propia

Estos datos se interpretaron con las aportaciones de Catach (1996), quien propone que pensemos la lengua en términos de lo que ella denomina "lengua prima" - en su teoría, propone que: "Todo lenguaje L provisto de un oral A y de un sistema de 
escritura desarrollado B se convierte en L', según la ecuación AxB=L>L')” (p. 310)—, pues en las sociedades actuales (desarrolladas) las personas deben establecer continuamente puentes entre el lenguaje oral y el lenguaje escrito, que interactúan y se complementan de manera constante. Sin embargo, en el caso bilingüe que se aborda, los puentes que se deben establecer son muchos: entre el popoluca (lengua extensamente dominante en la localidad de estudio) y el español oral, y entre sus versiones oral y escrita. Estos puentes se encontraron en todo momento: en el salón de clases, donde el popoluca es omnipresente, al leer un texto en español o al producir uno como parte de sus tareas extraescolares.

Con esto se evidencia que la colaboración con los intérpretes permitió el acercamiento a nuevos significados. El etnógrafo experimentó un cambio de perspectiva sobre el papel que cumplía el popoluca en el aprendizaje de la lengua escrita del español.

\section{Conclusiones}

Este trabajo permitió analizar un proceso colaborativo poco común para el contexto mexicano. Se conformó un equipo de trabajo investigador-intérpretes que hizo posible superar dilemas de orden ético, teórico y epistemológico. Como se pudo advertir, las tareas de traducción e interpretación requeridas, de por sí complicadas por la interacción entre oralidad y escritura, se dificultan aún más si se trata de intérpretes no profesionales (escasos en México cuando de lenguas indígenas hablamos). Esto hace necesario comprometerse en un proceso de construcción de roles, actividades o funciones, en el que hay que invertir tiempo yotros recursos (materiales y económicos). Además, se requiere desarrollar asesorías previas al trabajo de campo para que el investigador clarifique el objetivo de la investigación, contextualice el trabajo que se está desarrollando y capacite en el uso de los instrumentos de recolección de datos y en el manejo de equipo tecnológico que apoyan la labor del intérprete.

Sobre la marcha, es importante precisar las estrategias de trabajo partiendo de las dudas del intérprete. Se requiere diálogo cercano para afinar la toma de notas de campo, para ampliarlas y discutirlas, además de desarrollar reuniones informales al terminar cada uno de los eventos observados con el fin de aclarar las impresiones mutuas. En este caso, la intérprete que más colaboró en el estudio desempeñó un rol de mediadora lingüístico-cultural; sus intervenciones permitieron una mejor comprensión de los referentes culturales de los jóvenes y sus familias. Se generó un ambiente mucho más propicio para que los estudiantes expresaran sus ideas.

Se considera un logro para la investigación la construcción de las transcripciones de diversos eventos derivados de las clases de español, con las cuales se recuperó el popoluca de la Sierra. Identificamos que los estudiantes recurrían a la lengua indígena para comprender los contenidos de español, pues es una parte importante de sus herramientas de pensamiento. Todo esto fue posible gracias al trabajo desarrollado con los intérpretes. 
En este sentido, la investigación permitió visualizar que los docentes responsables de brindar atención educativa a estos jóvenes deben tener un conocimiento muy específico de la lengua de sus alumnos para saber cómo enfrentan ellos el aprendizaje, cómo interactúan sus conocimientos lingüísticos concretos con sus esfuerzos por aprender lo que deben aprender según la escuela. Hablamos de una didáctica adaptada a la población específica, lo que supone un conocimiento lingüístico y cultural, y por lo tanto una modificación efectiva del subsistema de enseñanza. Consideramos que estos son aspectos básicos por atender si queremos mejorar la atención educativa que reciben estos jóvenes.

\section{Referencias}

Bertely, M. (2005). Usos del español y la lengua indígena en los espacios escolares de educación primaria para niños y niñas migrantes. México: SEP.

Catach, N. (1996). La escritura en tanto plurisistema, o teoría de L prima. En N. Catach (comp.), Hacia una teoría de la lengua escrita (pp. 310-331). Barcelona: Gedisa.

Cummins, J. (2002). Lenguaje, poder y pedagogía. Niños y niñas bilingües entre dos fuegos. Madrid: Morata.

De Moura, C., Wolff, L. y García, N. (2001). Telesecundaria de México: televisión educativa en zonas rurales. Sustainable Development Technical Paper. Washington, DC: Inter-American Development Bank. Recuperado de: http:// enfoques.webs.com/rodolfo/Telesecundaria\%2oen\%2oMexico.pdf

Dolz, J., Gagnon, R. y Mosquera, S. (2009). La didáctica de las lenguas: una disciplina en proceso de construcción. Didáctica, lengua y literatura, (21), 117-141. Recuperado de: http://revistas.ucm.es/index.php/DIDA/article/view/ DIDA0909110117A

García, C. (2004). Traducir la oralidad: su incidencia en el proceso de aprendizaje de la traducción. Glosas didácticas, (13), 115-127. Recuperado de: http://www. um.es/glosasdidacticas/GD13/GD13_11.pdf

Giménez, C. (1997). La naturaleza de la mediación intercultural. Migraciones, (2), 125-159.

Hamel, R. (2003). El papel de la lengua materna en la enseñanza: Particularidades de la educación bilingüe. En I. Jung y L. López (coords.), Abriendo la escuela, lingüística aplicada a la enseñanza de lenguas (pp. 248-260). España: Morata.

Jiménez-Ivars, A. (2002). Variedades de interpretación: modalidades y tipo. Hermeneus: Revista de la Facultad de Traducción e Interpretación de Soria, (4), 1-15. Recuperado de: https://dialnet.unirioja.es/servlet/articulo?codigo=290509

Jiménez, Y. (2011). Exclusión, asimilación, integración, pluralismo cultural y "modernización" en el sistema educativo mexicano: un acercamiento histórico a las escuelas de educación pública para indígenas. CPU-e, Revista de Investigación Educativa, (12), 1-24. Recuperado de: http://www.uv.mx/cpue/ num12/inves/jimenez-exclusion-asimilacion.html

Lahire, B. (2010). La desigualdad ante la cultura escrita escolar: el caso de la “expresión escrita” en la escuela primaria. En J. Vaca (coord.), Prácticas 
de lengua escrita: vida, escuela, cultura y sociedad (pp. 121-146). Xalapa, México: Instituto de Investigaciones en Educación, Universidad Veracruzana, Biblioteca Digital de Investigación Educativa. Recuperado de: http://www. uv.mx/bdie/files/2012/10/vaca_practicas_lectura.pdf

Loredo, J., Alvarado, F., Frutos, V. y Mancera, E. (2006). Prácticas emergentes de la inserción de las computadoras en telesecundarias. México: CONAFE

Márquez, D. (2011). Las once competencias del traductor: El perfil ideal en servicios de traducción. Comunicación, Cultura y Política. Revista de Ciencias Sociales, (4), 53-68. Recuperado de: https://journal.universidadean.edu.co/ index.php/revistai/article/view/659

Podestá, R. (2003). Nuestros pueblos de hoy y siempre. El mundo de las niñas y los niños nahuas de México a través de sus propias letras y dibujos. México: Instituto de Ciencias Sociales y Humanidades-Benemérita Universidad Autónoma de Puebla

Rockwell, E. (2006). Apropiaciones indígenas de la escritura en tres dominios: religión, gobierno y escuela. Cultura Escrita y Sociedad, (3), 161-218.

Rockwell, E. (2008). Relaciones con la cultura escrita en una comunidad nahua a principios del siglo XX: temas recurrentes en los relatos orales. Revista Lingua Escrita, (4), 1-17.

Rockwell, E. (2009). La experiencia etnográfica. Historia y cultura en los procesos educativos. Argentina: Paidós.

Sales, D. (2003). Interacción comunicativa intercultural con inmigrantes procedentes de la cultura china. En Grupo CRIT (ed.), Claves para la comunicación intercultural (pp. 114-139). Castellón de la Plana: Publicacions de la Universitat Jaume I. Recuperado de: http://www.crit.uji.es/biblio/doraclaves.pdf

Universidad Veracruzana Intercultural (UVI) (2007). Licenciatura en Gestión Intercultural para el Desarrollo: programa multimodal de formación integral. Xalapa: Universidad Veracruzana. 